\title{
Table of Scottish Parliament Statutes
}

1999 Mental Health (Public Safety and Appeals) (Scotland) Act 26, 54

2000 Abolition of Feudal Tenure etc (Scotland) Act 274, 279, 299, 301 s1 ................................................... 280



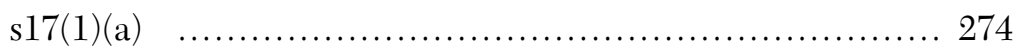

ss19-21 ................................................. 275

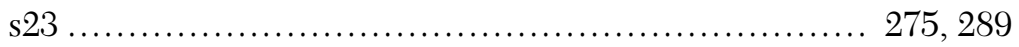

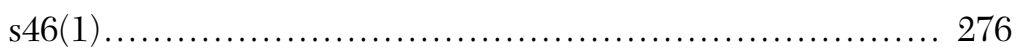



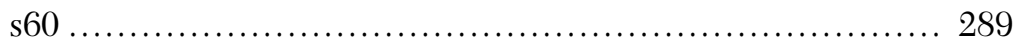

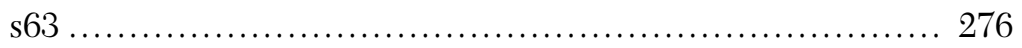

s63(2), (3) ........................................... 276

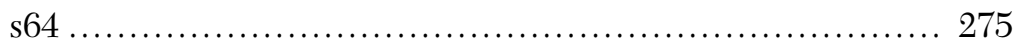

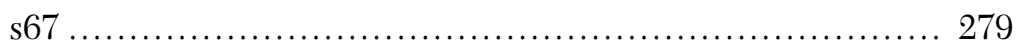



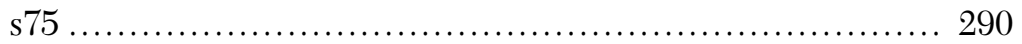



Adults with Incapacity (Scotland) Act .................... 72

Bail, Judicial Appointments etc (Scotland) Act

Pt 1 .................................................. 212

Education and Training (Scotland) Act ................... 177

Ethical Standards in Public Life etc (Scotland) Act .......... 132

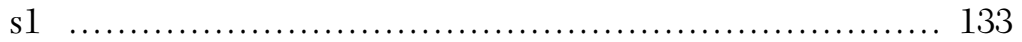

s5

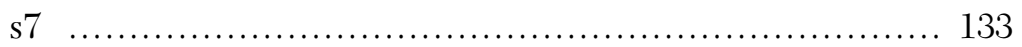

s9

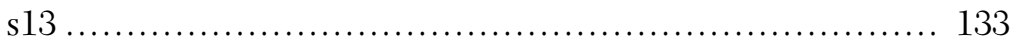

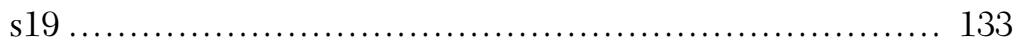

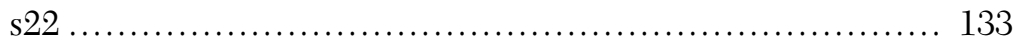


s34

72,172

s36(a), (b) 172

National Parks (Scotland) Act 327

s1 324,328

sch 1 para 3 328

Standards in Scotland's Schools etc Act $94,97,160,161,171$ s1 158

s2 158

s2(1) 159

$\mathrm{s} 2(2)$ 159,160

$\mathrm{s} 2 \mathrm{~A}$ 163

s3(1), (2) 161

s4 161

s5(1)

94, 159

s5(2)

s5(2)(c) 94 s5(4) 94

s6 161

s6(1) 159

s7 161

s9 161

s10 161

s10A 162

s10B 162

s10C 162

s12. 161







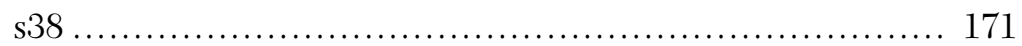

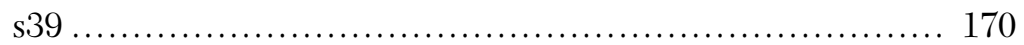

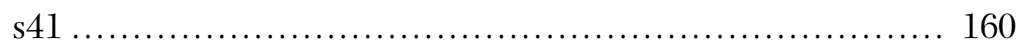

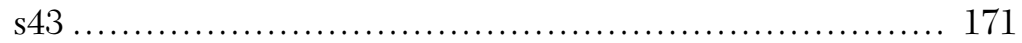

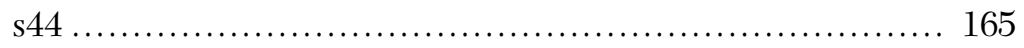

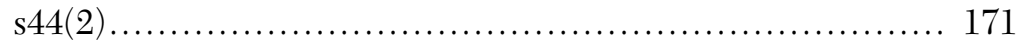

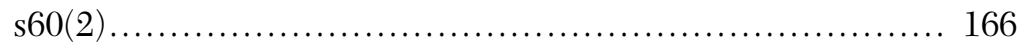

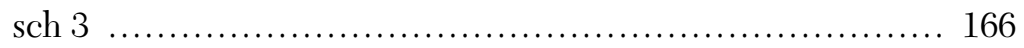

2001 Abolition of Poinding and Warrant Sales Act .................. 24

Convention Rights (Compliance) (Scotland) Act ............ 54 
s10 216

Education (Graduate Endowment and Student Support)

(Scotland) Act 178 $\mathrm{s} 1$ 178

s4 178

Housing (Scotland) Act $72,141,150$

Pt 2 145

Pt 3 150

s22 145

ss $34-37$ 146

s43 155

sch 3 para $2(1)($ a) 145

$\operatorname{sch} 4$ 152

International Criminal Court (Scotland) Act

s1 219

s4 219

Leasehold Casualties (Scotland) Act ....................... 279

ss2-4 .................................................. 279

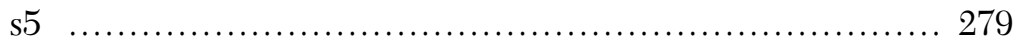

s6

s7

Mortgage Rights (Scotland) Act ..................... 72, 280

Protection from Abuse (Scotland) Act........................ 72

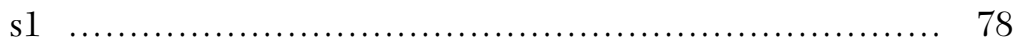

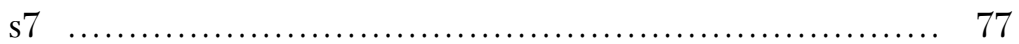

Regulation of Care (Scotland) Act

s52(1)(a), (b) ............................................. 221

Salmon Conservation (Scotland) Act ................. 217, 327

Transport (Scotland) Act ............ 343. 344, 347, 349, 350, 360

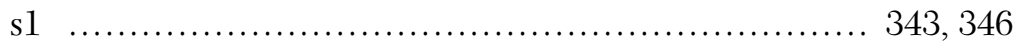

s3

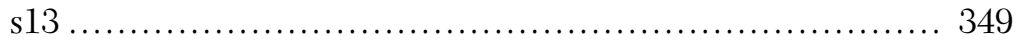

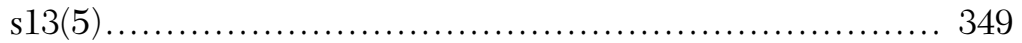

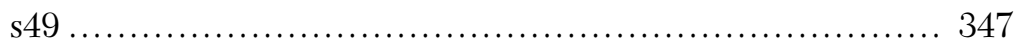

s49(3) .................................................... 347

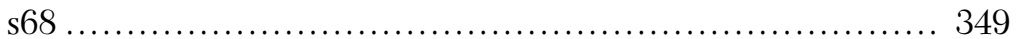

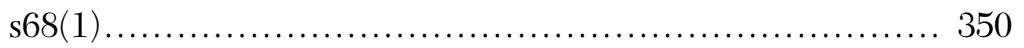

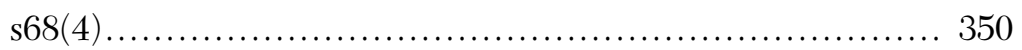

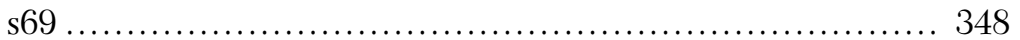


2002 Debt Arrangement and Attachment (Scotland) Act

Education (Disability Strategies and Pupils' Educational Records) (Scotland) Act .............................. 179 s1

s4 179

Fur Farming (Prohibition) (Scotland) Act $216,217,327$

Marriage (Scotland) Act 70

Protection of Wild Mammals (Scotland) Act ... 54, 216, 217, 327 Schools Education (Amendment) (Scotland) Act s1(1) 171

Scottish Local Government (Elections) Act 135 Scottish Public Services Ombudsman Act 134,135 s15(1) 134 s16(3) 134 sch 2 Pt 3 para 90 178

$\operatorname{sch} 6$ 134

Scottish Qualifications Authority Act 172 s1

s3 172

Sexual Offences (Procedure and Evidence) (Scotland) Act 54, 251,

Water Industry (Scotland) Act

s51 324

s56(1)(aa) ........................................... 336

2003 Agricultural Holdings (Scotland) Act ....................... 72 s18(4) ................................................... 327

Building (Scotland) Act



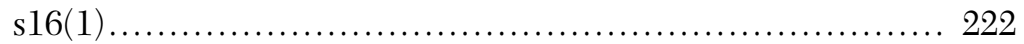

s16(1)(b) f......................................... 222

s19(4)(b) ............................................. 222

s20(1)(b) ............................................ 222

s34(2) .................................................. 222

s37(4)(c) …....................................... 222

Commissioner for Children and Young People (Scotland) Act 61

Criminal Justice (Scotland) Act ........................... 209 


\section{Pt 2}

213

$\operatorname{s14}(2)$ 214

s16(1) 214

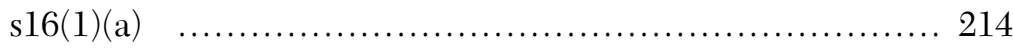

s17 ................................................... 214

s19 ................................................... 217

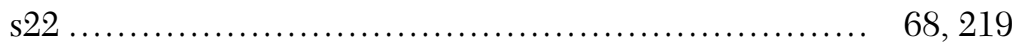

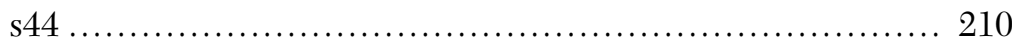

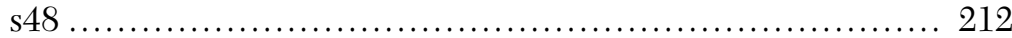

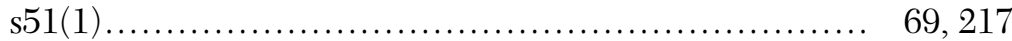

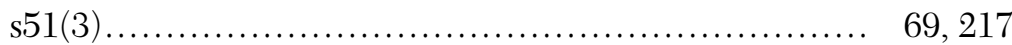



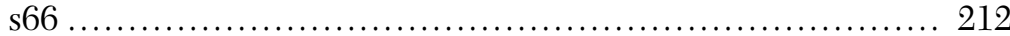

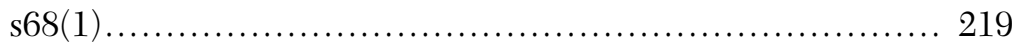

s74 ................................................... 216

Dog Fouling (Scotland) Act ........................ 216, 326

Education (School Meals) (Scotland) Act ...............24, 163-4

Homelessness etc (Scotland) Act..................... 146, 148

Land Reform (Scotland) Act $\ldots \ldots \ldots \ldots \ldots \ldots \ldots$ 19, 217-18, 329

Pt 2 .................................................. 325



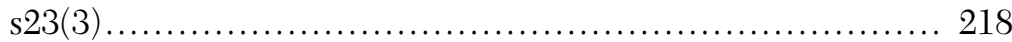

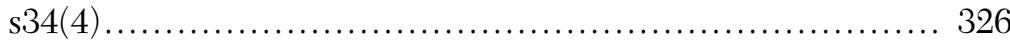

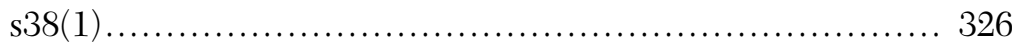

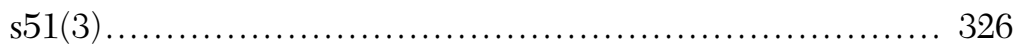

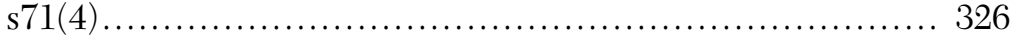

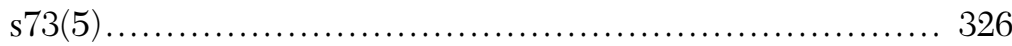

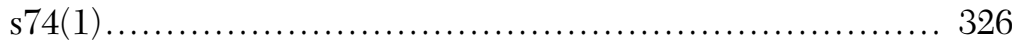

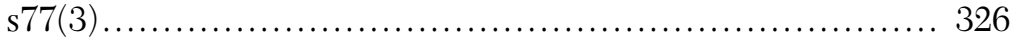

Local Government in Scotland Act ....................... 137

s1(2) f................................................ 137

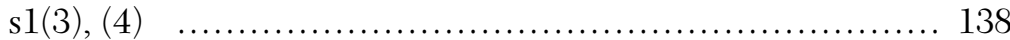

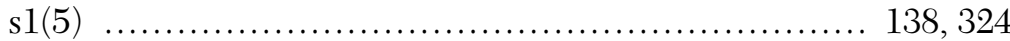

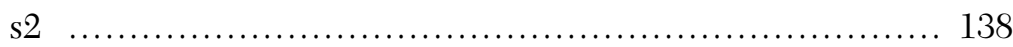

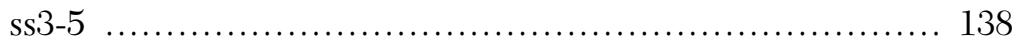

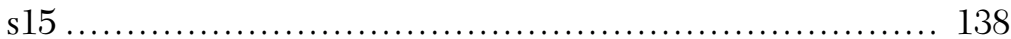

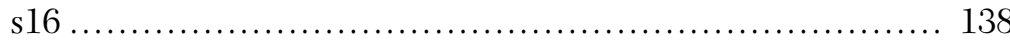

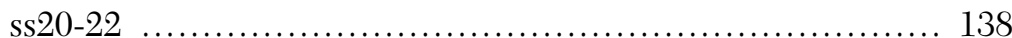

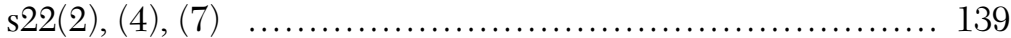


s26

s34 327

Mental Health (Care and Treatment) (Scotland) Act .... 72

s318 222

s328 257

National Galleries of Scotland Act 86

Protection of Children (Scotland) Act .... 68 s3 217

Robin Rigg Offshore Wind Farm (Navigation and Fishing)

(Scotland) Act 217,326

Salmon and Freshwater Fisheries (Consolidation)

(Scotland) Act ............................. 217, 327, 339

Title Conditions (Scotland) Act .......... 274, 277, 280, 299, 301

Pt 1 281,282

Pt 2 284

Pt 3

285,286

Pt 6 290

Pt 7 291

Pt 8 292

Pt 9 292

s1 282

s1(1) 285

s3(6) 282

$\mathrm{s} 4(2)(\mathrm{c})$ 282 s4(5) 282

s5 282

s8(1) 282

s8(3) 282,285

s9 283

s10 283

s10A 283

ss11-13 283

s14 283



$\mathrm{s} 15$ 284

s16 284

ss21-24 284

ss26-31 284

ss $32-34$ 285 


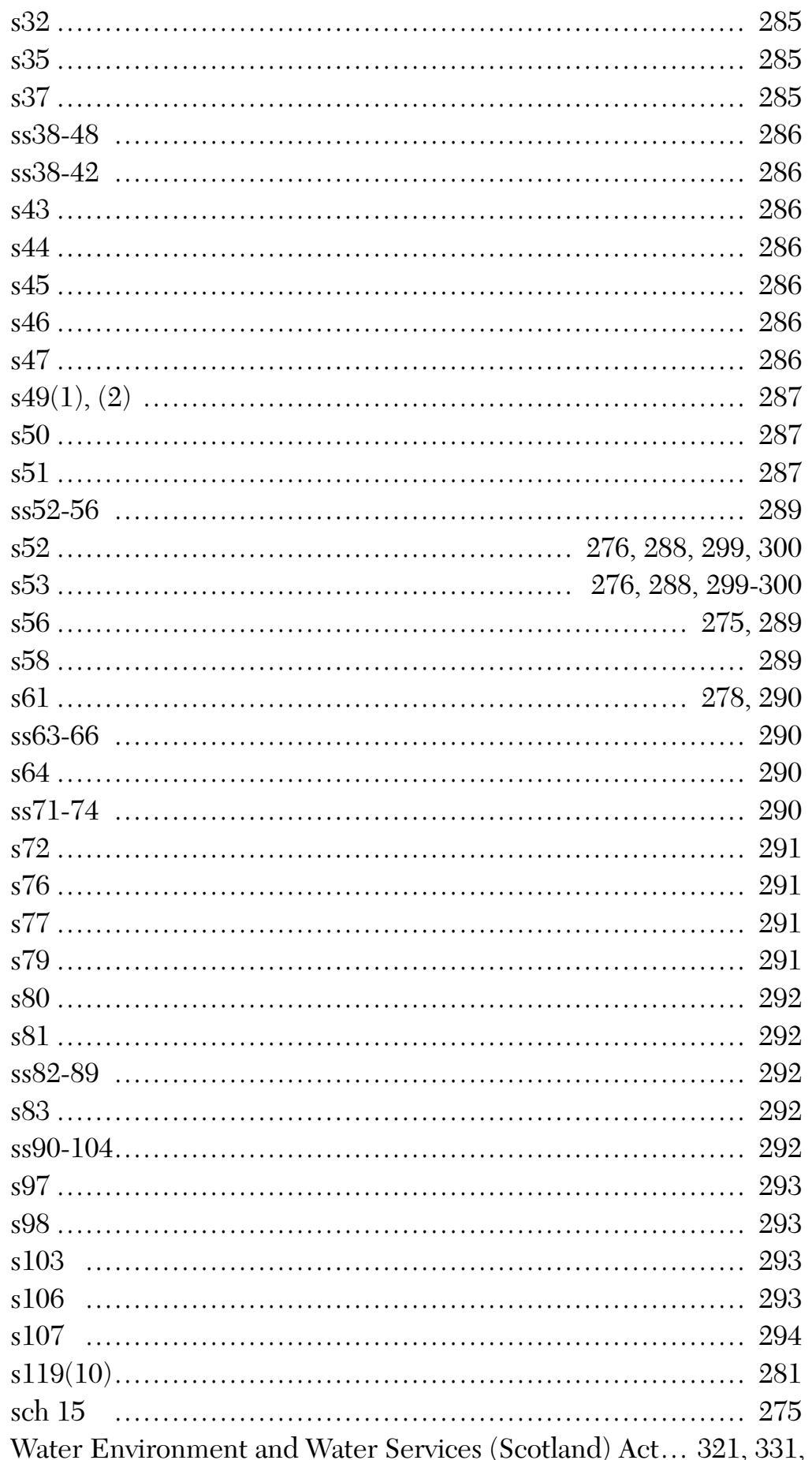






2004 Antisocial Behaviour etc (Scotland) Act ... 149, 227, 240, 243, 244 Pt 9 ................................................. 241

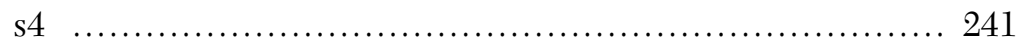



s4(2)(a) .................................................. 245



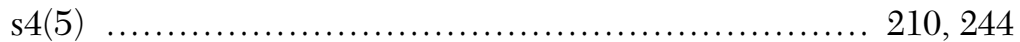

s9(1) f............................................ 210, 244

s9(2)(b) f............................................. 210

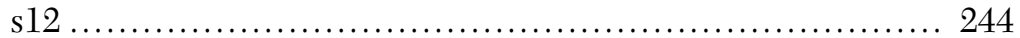

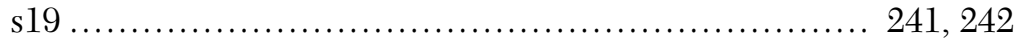



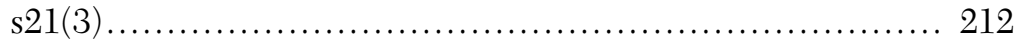

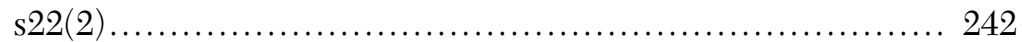

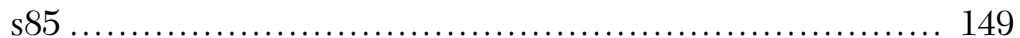

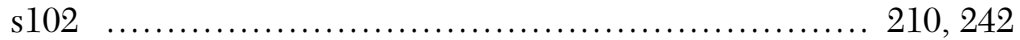





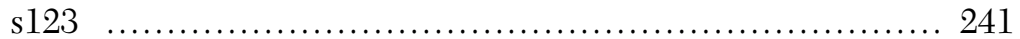





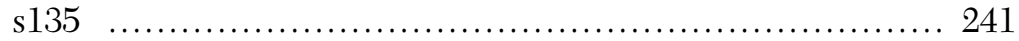

s135(1), 2) ............................................... 245

Criminal Procedure (Amendment) (Scotland) Act

Pt 2 ................................................... 212

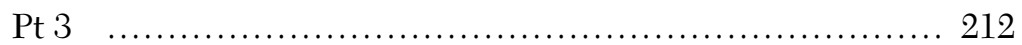

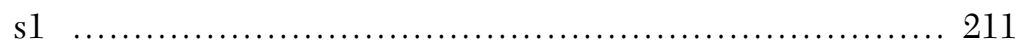

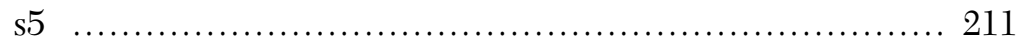





Education (Additional Support for Learning)

(Scotland) Act .................................. 171, 173-5

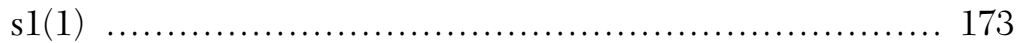






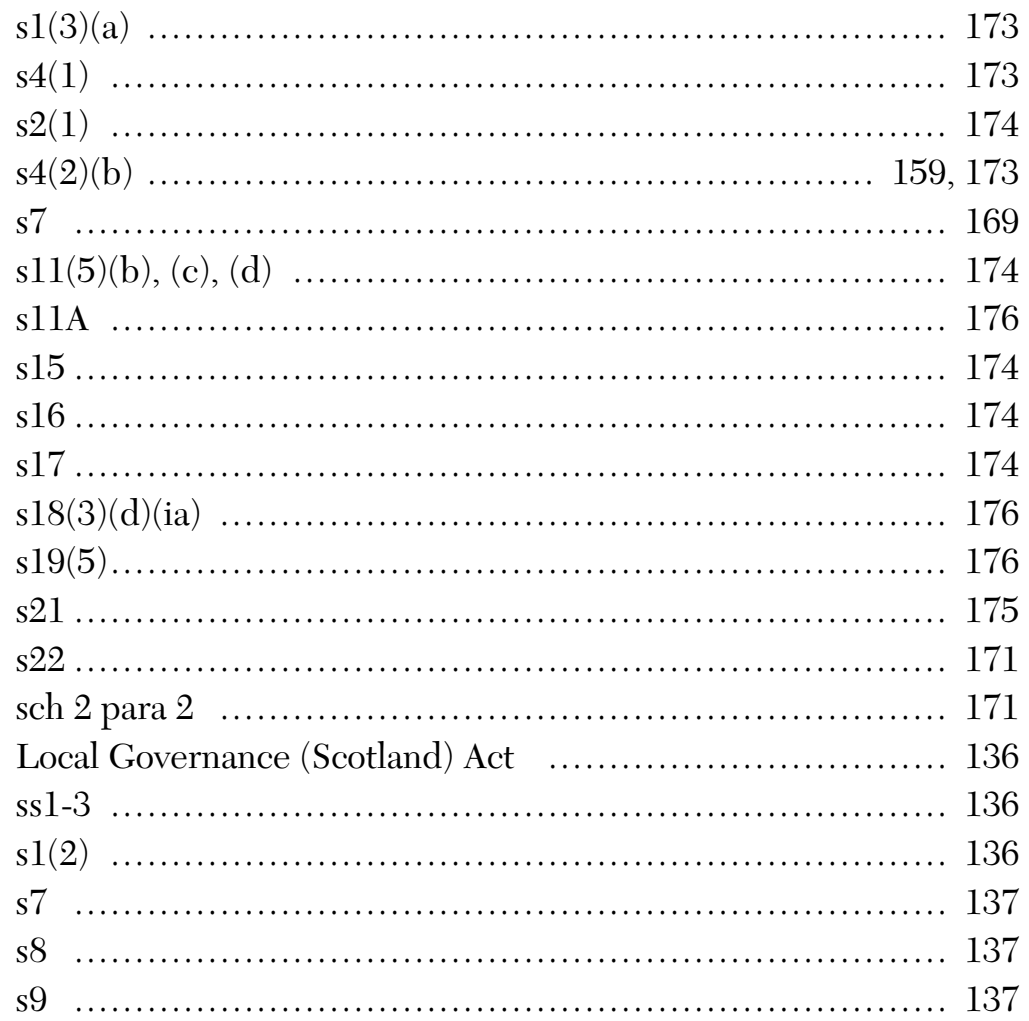

Nature Conservation (Scotland) Act $\ldots \ldots \ldots \ldots$ 217, 319, 328, 329

s1 ................................................... 329



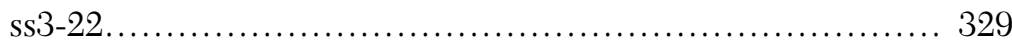

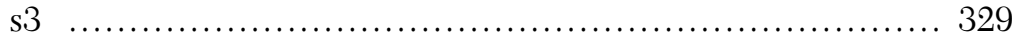

ss29-37 .................................................. 329

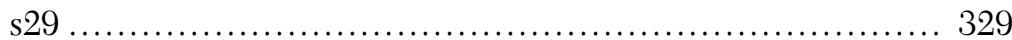



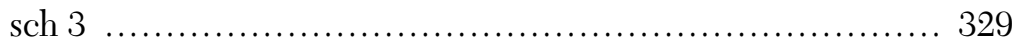

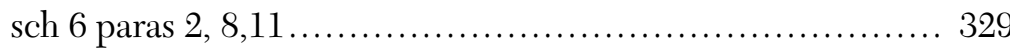



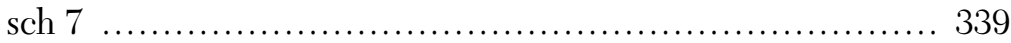



School Education (Ministerial Powers and Independent

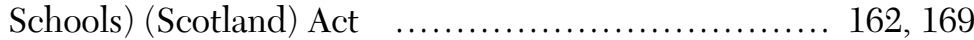

s1 .................................................... 162

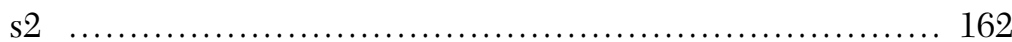




Tenements (Scotland) Act 274, 294-8, 301 ss $1-3$ 295

s2(3), (4) 295

s2(6), (7) 295

s.3 295

ss4-6 296

s7 296

ss $8-10$ 296

s12 297

s17 297

s18 297

s19 297

s20 297

s21 297

s22 297

s22(3) 298

s24 298

sch 1 296

sch 3 298

Vulnerable Witnesses (Scotland) Act $214,251,255-62$ $\operatorname{s11}(1)(\mathrm{a})$ 256

$\mathrm{s} 11(1)(\mathrm{b})$ 257

$\operatorname{s11}(2)(a),(b),(c),(d),(e),(f)$ 257 s11(4).... 256

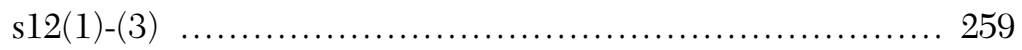

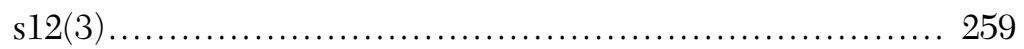

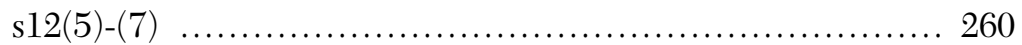

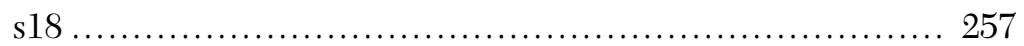

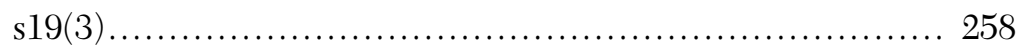

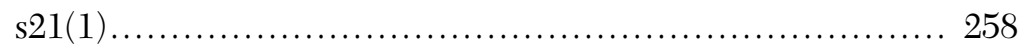



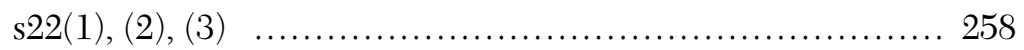

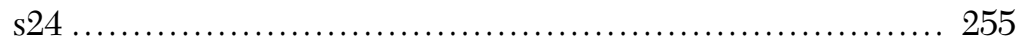

2005 Breastfeeding etc (Scotland) Act ................... 216, 221

Charities and Trustee Investment (Scotland) Act 103-4, 122, 169,

Pt 1 





s34

ss $39-43$

110,113

ss44-48 110,113

ss49-64 110, 113

ss66-70 110

s71(a).... 120

ss75-78 110

s75 110

s76 120

s78 120

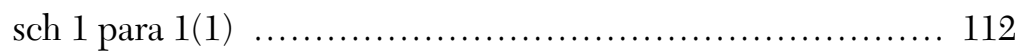

para $3(1)(a),(b)$ 112

Emergency Workers (Scotland) Act 216 s1 220

$\operatorname{sl}(1)$ 216

s2 220

$\mathrm{s} 2(3)$ 220

s3 220

s4 221

s5 220

s6 220

Environmental Assessment (Scotland) Act $318,321,330$ ss4-10 322

ss $4-6$ 331

sch 2 para 1 319

Fire (Scotland) Act 221

s39 216

s74(4)(b) 221

Further and Higher Education (Scotland) Act 176 s3 176

$\mathrm{s} 4$ 176

s14 177

s20 324

s20(1)(a), (b), (c) ....................................... 177

s20(6) 177

s20(2)(a), (b) ........................................... 177

s20(3)(a), (b) .......................................... 177



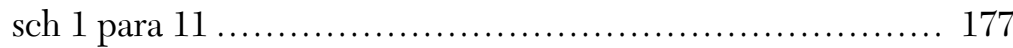






Smoking, Health and Social Care (Scotland) Act

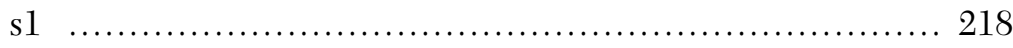

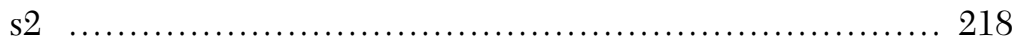

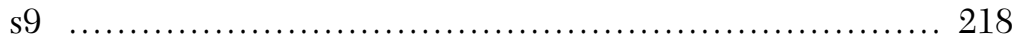

Transport (Scotland) Act ............ 140, 319, 344, 349, 350, 360

Pt 1 ch 1 ............................................... 346

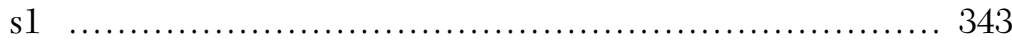



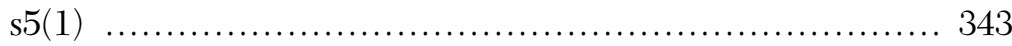

s5(2) f.............................................. 319, 345

s40 ................................................... 349

Water Services etc (Scotland) Act ........................ 332

2006 Animal Health and Welfare (Scotland) Act.............. 216, 217

Family Law (Scotland) Act .................... 75, 76, 78, 80 
s1 70

s2 70

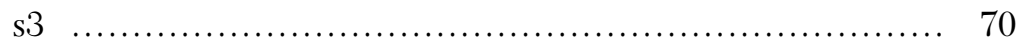



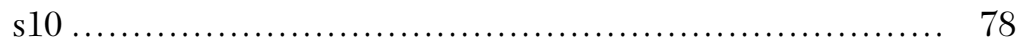

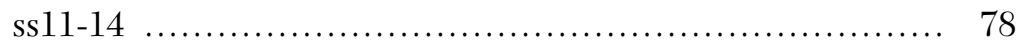

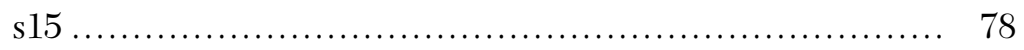

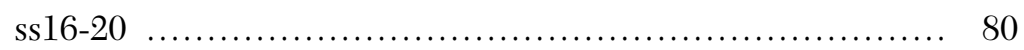





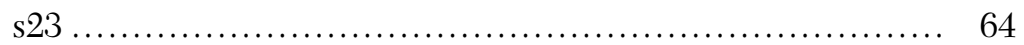

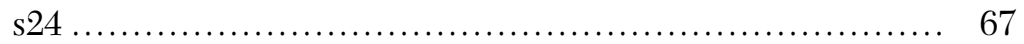

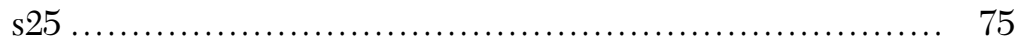

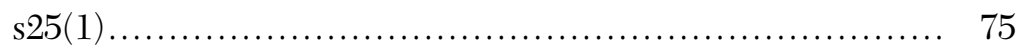

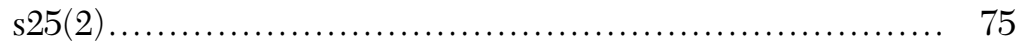

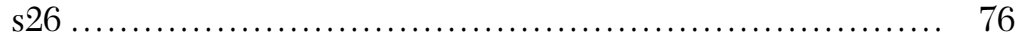

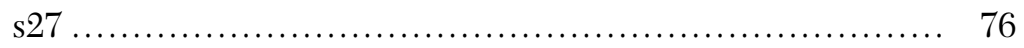

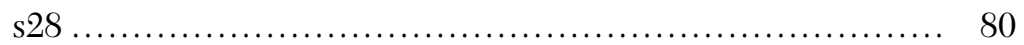

s28(2)(a), (b), (c) ......................................... 80

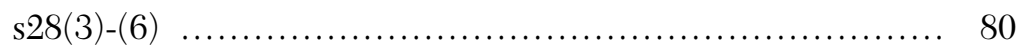

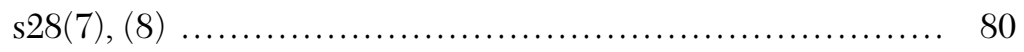



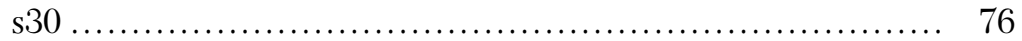



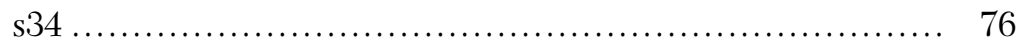

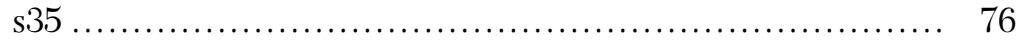

Housing (Scotland) Act $\quad \ldots \ldots \ldots \ldots \ldots \ldots \ldots . . \ldots 148,152,153,216$

s14 ....................................................... 152

Local Electoral Administration and Registration Services

(Scotland) Act ......................................... 218

Planning etc (Scotland) Act ....................... 218, 325, 327

s2 .................................................... 324

Police, Public Order and Criminal Justice (Scotland) Act

s51(2) .................................................... 210

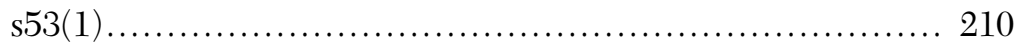

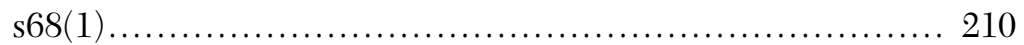

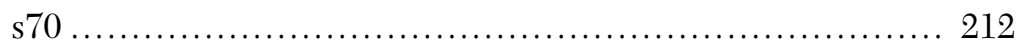






s81(3) 213

s81(6) 213

s82 213

s83 213

s84 213

Senior Judiciary (Vacancies and Incapacity) (Scotland)

Act (asp 9) 196

Local Electoral Administration and Registration Services

(Scotland) Act ......................................... 63

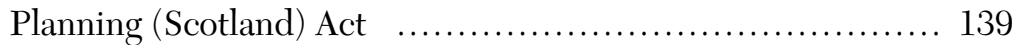

Schools (Health Promotion and Nutrition) (Scotland) Act $\quad . \quad 163$ s1 163

Scottish Commission for Human Rights Act ................ 51

Scottish Schools (Parental Involvement) Act ................ 166

s1 .................................................... 166

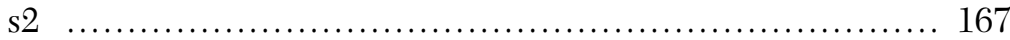

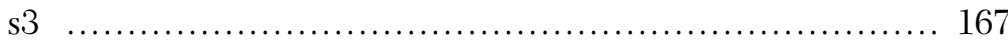

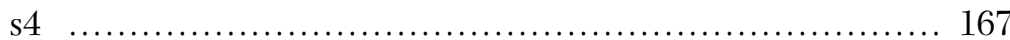

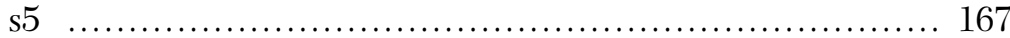



Waverley Railway (Scotland) Act

$\operatorname{s1}(3)$ 359

s48 359

2007 Adoption and Children (Scotland) Act ................. 63, 75

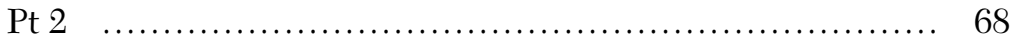

Pt 4 ................................................. 209

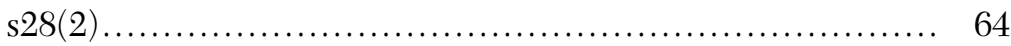

s29(3) ............................................ 64, 76

Airdrie-Bathgate Railway and Linked Improvements Act s47 359

Aquaculture and Fisheries (Scotland) Act 217,327 s2 218

Bankruptcy and Diligence (Scotland) Act ............. 76, 304 Pt 2 ................................................... 304

Christmas Day and New Year's Day Trading (Scotland) Act ... 216

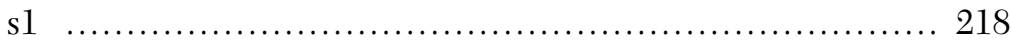

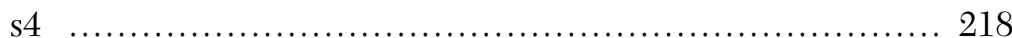

Criminal Proceedings etc (Reform) (Scotland) Act ......... 54 


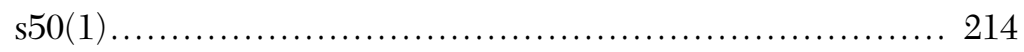

s50(1)(a)(iv) ......................................... 214

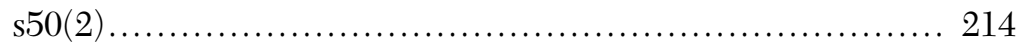

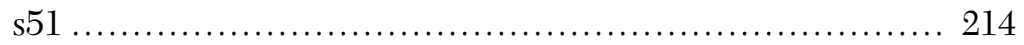

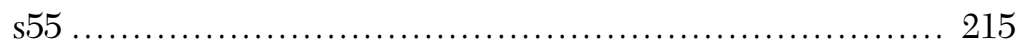

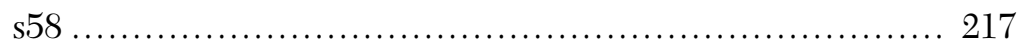

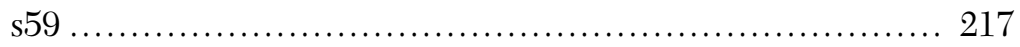

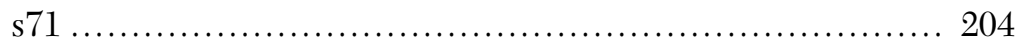

Crofting Reform etc Act

s28

Custodial Sentences and Weapons (Scotland) Act s63

Edinburgh Airport Rail Link Act

s51 359

Glasgow Airport Rail Link Act s46 359

Prostitution (Public Places) Scotland) Act 216 s1

Protection of Vulnerable Groups (Scotland) Act $\quad \ldots \quad$ 68, 216, 217 Schools (Health Promotion and Nutrition) (Scotland)

Transport and Works (Scotland) Act

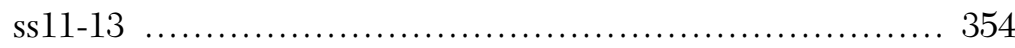

s13 
2008 Abolition of Bridge Tolls (Scotland) Act ........... 323, 337, 348

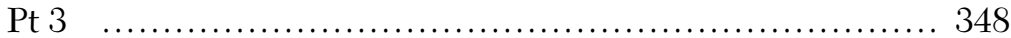

Glasgow Commonwealth Games Act ...................... 323

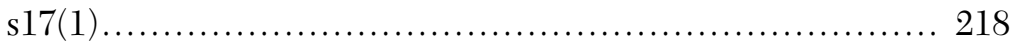

Graduate Endowment Abolition (Scotland) Act............. 178

Judiciary and Courts (Scotland) Act $\quad 183,190,192,206,212,323$

s1 ................................................... 192

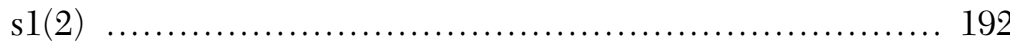

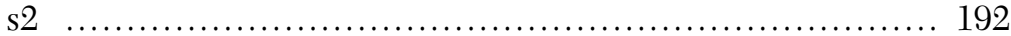



ss4-8 .................................................. 196

ss9-18................................................. 192



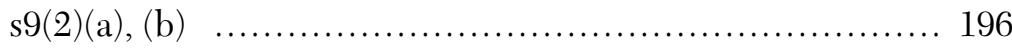

s9(3) .................................................... 196

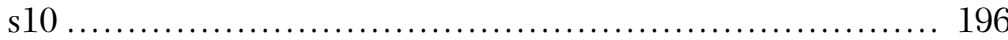

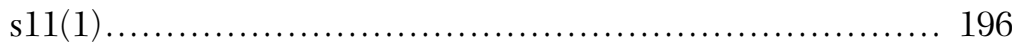

s12 f.............................................. 193, 197

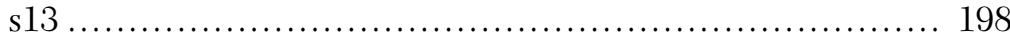



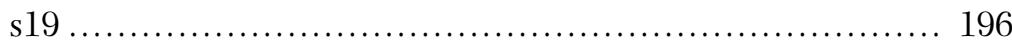

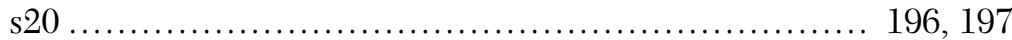

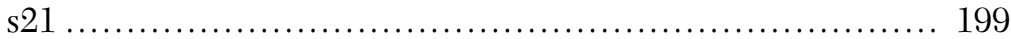



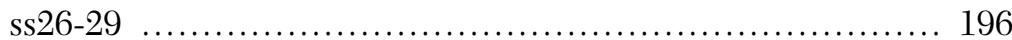

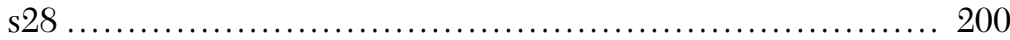

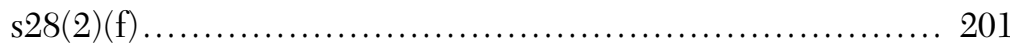



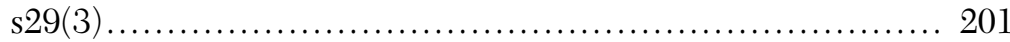





s30(5) ................................................ 202

s31 f.................................................. 202



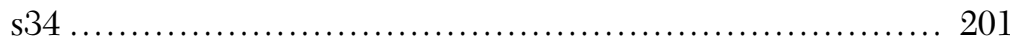

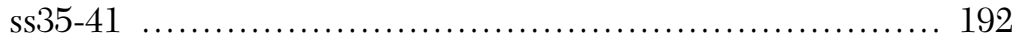

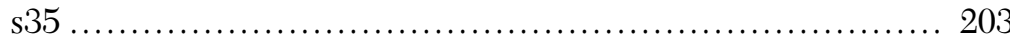

s35(1), (2), (3), (4) ...................................... 203

s35(6), (7), (8), (9), (10), (11) .......................... 203 


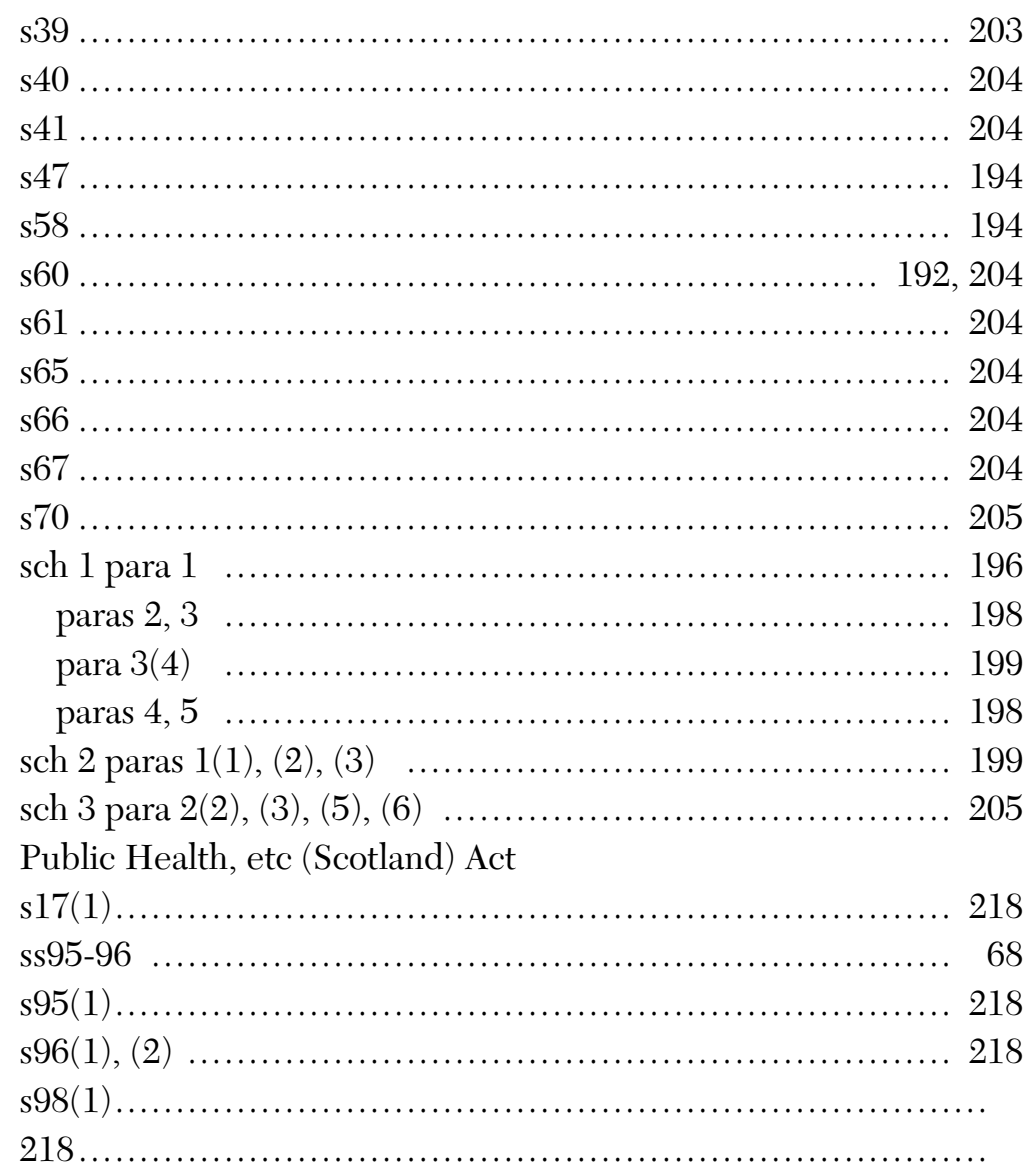

Scottish Register of Tartans Act ..................... 86, 323



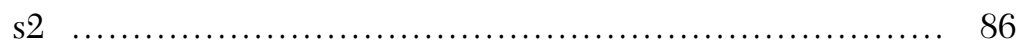

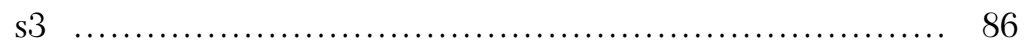

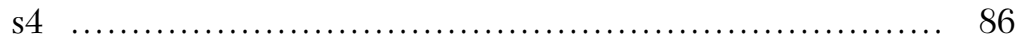

2009 Climate Change (Scotland) Act ..... 208, 318, 323, 324, 326, 334,

335,338

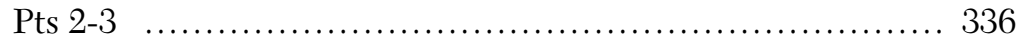

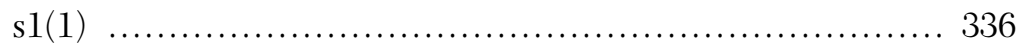

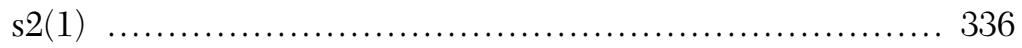



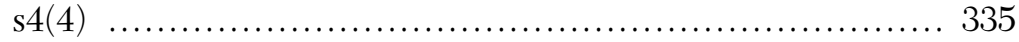





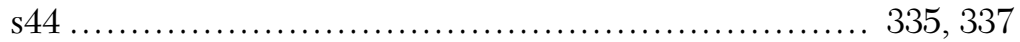




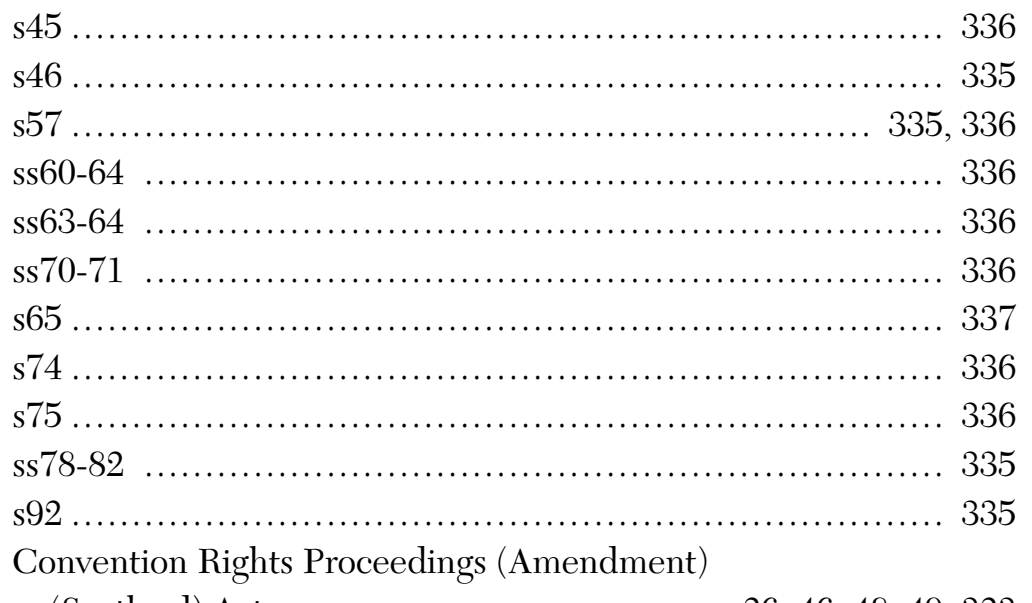

(Scotland) Act ............................ 26, 46, 48, 49, 323

Damages (Asbestos-related Conditions) (Scotland) Act........ 54 Education (Additional Support for Learning) (Scotland) Act 175

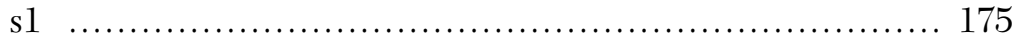



s1(8)(b) f........................................... 176



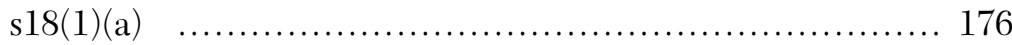

s21 .................................................. 176

Flood Risk Management (Scotland) Act ........ 321, 323, 332, 333

s1(1), (2) …...................................... 333

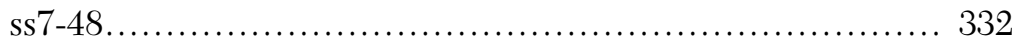

s27(4) ................................................ 324

Health Boards (Membership and Elections) (Scotland) Act ... 323

Offences (Aggravation by Prejudice) (Scotland) Act ..... 216, 323

s1 ................................................... 216

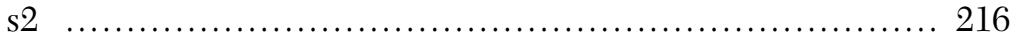

Public Services Reform (Scotland) Bill, SP Bill 26 ........... 101

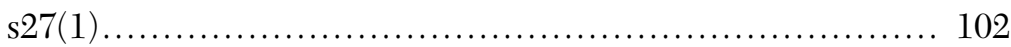

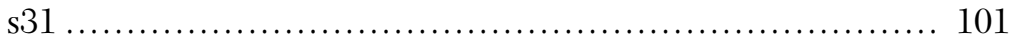

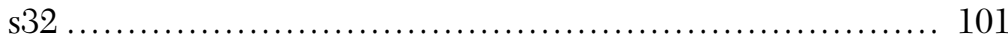

Scottish Local Government (Elections) Act …............. 135

Sexual Offences (Scotland) Act ......... 216, 218, 222, 223, 323



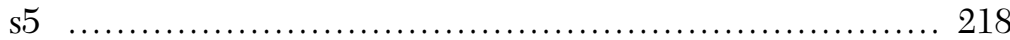

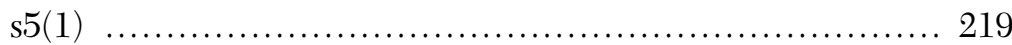

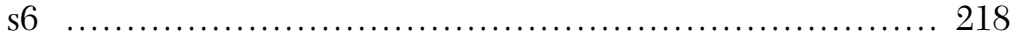




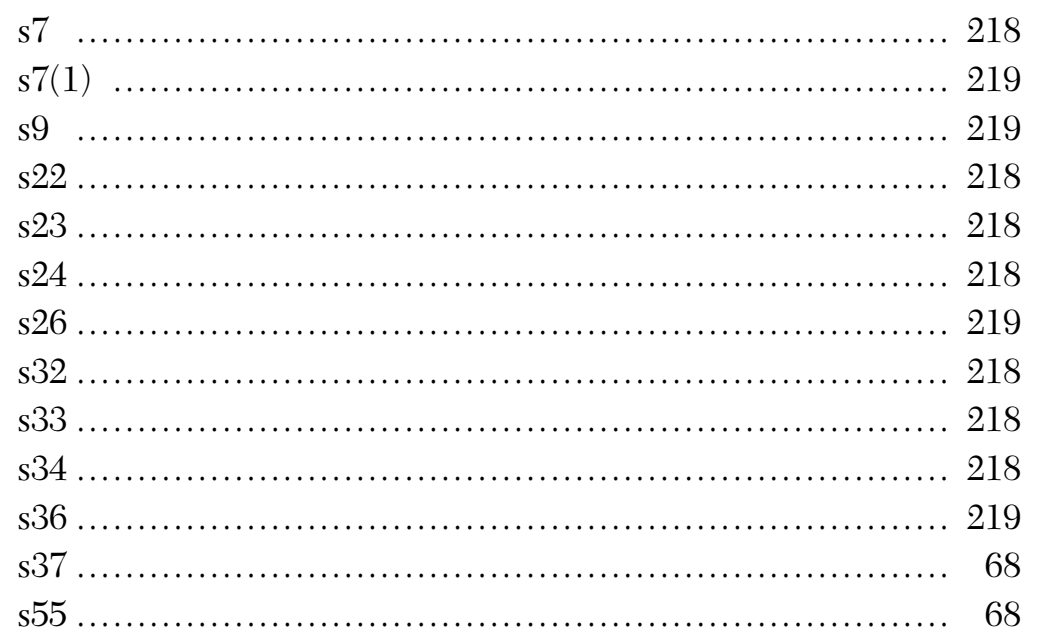

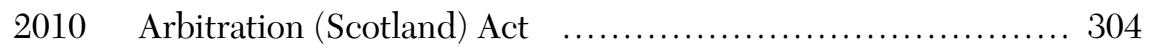

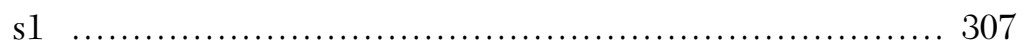

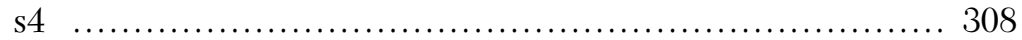

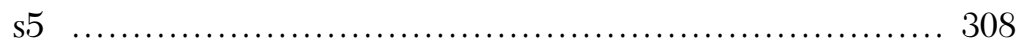

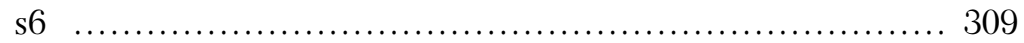

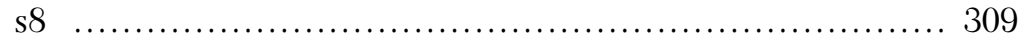

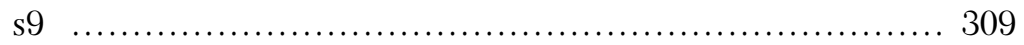

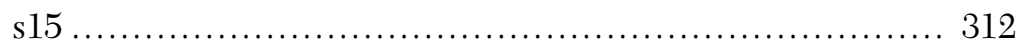

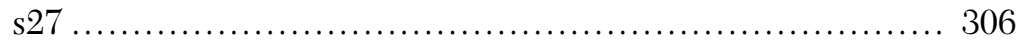

sch 1 (SA Rules) ............................... 306, 309-15

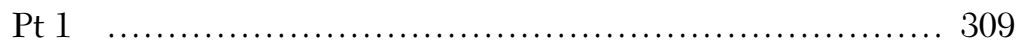

Pt 2 ................................................... 309

Pt 3 ................................................... 312

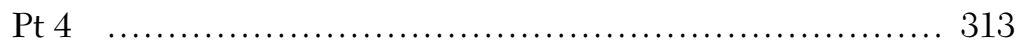

Pt 5 ................................................... 313

Pt 8 ................................................. 313

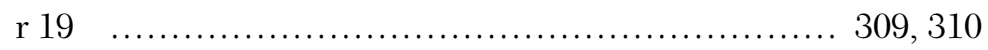

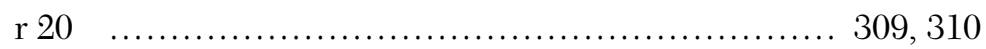

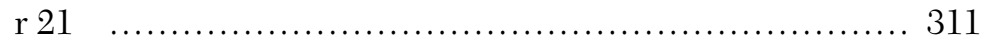

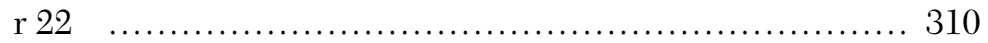

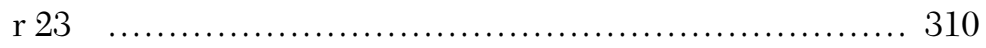

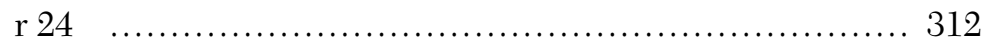

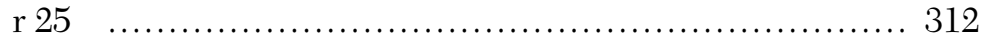

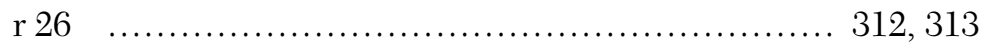

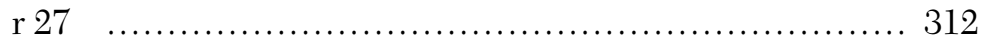

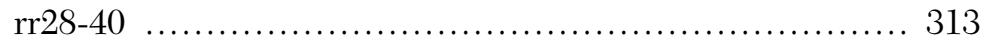






